\title{
Lytic Metastatic Bone Lesion
}

National Cancer Institute

\section{Source}

National Cancer Institute. Lytic Metastatic Bone Lesion. NCI Thesaurus. Code C35371.

A radiologic finding indicating the presence of bone destruction that results from the spread of cancer to the bone(s). 